\title{
Higher mortality for weekend admissions: quality of care or selection bias?
}

\author{
To the Editor:
}

We read the article by SuISSA et al. [1] with great interest. The authors retrospectively looked at all hospitalisations for chronic obstructive pulmonary disease (COPD) exacerbation or pneumonia during the period 1990-2007 from the healthcare databases of Quebec, Canada, and found higher mortality for weekend, but not Friday, admissions, relative to Monday-Thursday admissions. Similar weekend effects were observed for multiple medical conditions across different settings [2-4]. The increased mortality for weekend acute admissions is often attributed to lower quality of care during the weekend even though it could be equally plausible that sicker people came into hospital at weekends [2].

SuIsSA et al. [1] tried to control the differences in patient profiles by adjusting for age, sex, calendar year of admission, number of prior admissions and comorbidities, but they came short of specifically examining any clinical severity indicator at the time of admission. The concern over selective admission of sicker patients during the weekend may well be highlighted by the $25 \%$ lower number of admissions for COPD exacerbation or pneumonia on Saturday and Sunday ( $\sim 50000$ person-days) as compared with weekdays, including Friday (600 000 person-days), as shown in Table 2 of the original paper. For COPD exacerbation and pneumonia, there is no good reason to expect major variations in their real incidence by days of the week even if reduced traffic-related air pollution over the weekend is taken into account. The weekly rhythm of human activity is likely to impact illness behaviour: less ill patients tend to wait until the next weekday when healthcare is more accessible. This could well explain why the observed variations in the denominators exactly paralleled and dwarfed the marginal differences (a few per cent) in mortality rates (higher during Saturday and Sunday, but with no difference between Friday and other weekdays). Similar variations in the denominators were also present and apparently unaddressed in two other studies cited above $[3,4]$. Closer examination of the relevant denominators might well be called for in future studies.

None of the above three studies $[1,3,4]$ specifically examined variations in access to specialised procedures or sophisticated care across the week, which might otherwise have allowed attribution of the higher mortality of weekend admissions to poorer quality of care on Saturdays and Sundays. While it may be difficult to highlight any particular specialised procedures that could substantially impact mortality in the first-line care of COPD exacerbation and pneumonia, early endoscopy may be a candidate for cases of upper gastrointestinal bleeding. Using the 2004 Nationwide Inpatient Sample in the USA, patients with acute non-variceal upper gastrointestinal haemorrhage admitted at weekends had higher adjusted in-hospital mortality and were less likely to undergo early endoscopy within 1 day of hospitalisation than those admitted on weekdays [5]. However, patients with acute variceal haemorrhage admitted to non-teaching hospitals at weekends also had lower utilisation of early endoscopy, but there was no difference in survival. Among patients with peptic ulcer bleeding admitted to a teaching hospital capable of offering therapeutic endoscopy within 1 day in Hong Kong, Sunday or holiday admission did not increase the mortality risk, even though the waiting time for endoscopy was correlated with the risk of 30-day mortality [6]. Thus, access to specialised care could vary between different facilities and it may or may not be associated with increased mortality.

SuISSA et al. [1] also found a higher mortality during weekend and Friday stays, independent of the day of admission. However, mortality by the day of stay could conceivably be confounded by the day of admission for acute exacerbations and pneumonia if a considerable proportion of deaths occurred within $48 \mathrm{~h}$ of admission [2]. Selective discharge of more stable patients before the weekend, a rather common clinical practice, might also have contributed. Indeed, despite the normal rates of admission up until Friday, there were fewer patient-days over Saturday and Sunday, suggesting increased rates of discharge before the weekend [1]. Patients were at higher risk of dying on weekends and public holidays as compared with working days in a palliative care unit in Germany, even though reduced access to sophisticated levels of care was unlikely to be a major contributing factor for this particular patient group [7]. Again, closer examination of the profile of patients staying over the weekend would be required to delineate the actual cause(s).

In summary, the rhythm of human activity across the week, on both the side of the patient and the provider, might have impacted the observed weekend mortality. Further studies focusing on variations in the profiles 
of patients admitted and/or staying on different days would be required to delineate the cause(s). Premature attribution to reduced quality of care over the weekend may well run a risk of aggravating the delay in seeking care on the patient side.

0 @ERSpublications

The rhythm of human activity across the week might impact the observed increase in weekend hospital mortality http://ow.ly/KQm2g

Chi Chiu Leung, Lai Bun Tai, Irene Yip and Cheuk Ming Tam

Tuberculosis and Chest Service, Dept of Health, Hong Kong, China.

Correspondence: Chi Chiu Leung, Tuberculosis and Chest Service, Dept of Health, Wan Chai Chest Clinic, 99, Kennedy Road, Wan Chai, Hong Kong, China. E-mail: cc_leung@dh.gov.hk

Received: Feb 022015 | Accepted: Feb 042015

Conflict of interest: None declared.

\section{References}

1 Suissa S, Dell'Aniello S, Suissa D, et al. Friday and weekend hospital stays: effects on mortality. Eur Respir J 2014; 44: 627-633.

2 Calverley PM. Something better for the weekend? Eur Respir J 2014; 44: 568-569.

3 Barba R, Zapatero A, Losa JE, et al. The impact of weekends on outcome for acute exacerbations of COPD. Eur Respir J 2012; 39: 46-50.

4 Bell CM, Redelmeier DA. Mortality among patients admitted to hospitals on weekends as compared with weekdays. N Engl J Med 2001; 345: 663-668.

5 Ananthakrishnan AN, McGinley EL, Saeian K. Outcomes of weekend admissions for upper gastrointestinal hemorrhage: a nationwide analysis. Clin Gastroenterol Hepatol 2009; 7: 296-302.

6 Tsoi KK, Chiu PW, Chan FK, et al. The risk of peptic ulcer bleeding mortality in relation to hospital admission on holidays: a cohort study on 8,222 cases of peptic ulcer bleeding. Am J Gastroenterol 2012; 107: 405-410.

7 Voltz R, Kamps R, Greinwald R, et al. Silent night: retrospective database study assessing possibility of "weekend effect" in palliative care. BMJ 2014; 349: g7370.

\section{The emerging role of the contractile and vascular reserves in pulmonary arterial hypertension}

To the Editor:

We read with great interest the recent article by SHARma et al. [1] on the evaluation of right ventricular (RV) reserve in pulmonary arterial hypertension (PAH) by the dobutamine stress test. Their work highlights the potential clinical value of the RV systolic reserve into the evaluation of PAH patients beyond resting measurements. SHARMa et al. [1] reported that RV contractile reserve (tricuspid annular plane systolic excursion (TAPSE) and tricuspid annulus systolic myocardial velocity $\left(S^{\prime}\right)$ ) is markedly attenuated in PAH patients and correlates with exercise capacity. Although the merit of the work is in assessing RV contractile reserve by direct functional parameters, there are some topics that deserve discussion.

Stressors (exercise or pharmacological) of the RV-pulmonary circulation unit in patients with PAH assess the ability of the pathological RV to increase its output (contractile reserve) and of the remodelled pulmonary arterial system to accommodate the flow increase (vascular reserve) [2]. Dobutamine is an ino-chronotropic drug that, at low doses (up to $10-15 \mu \mathrm{g} \cdot \mathrm{kg}^{-1} \cdot \mathrm{min}^{-1}$ ), increases pulmonary blood flow passively without intrinsic vasomotor effects on the pulmonary circulation. The use of higher peak doses of dobutamine could have increased the vasomotor tone of the pulmonary circulation and could explain the significant increase in systolic pulmonary arterial pressure seen in controls [3]. The similar heart rate stress increase in PAH patients compared to controls reported by SHARMA et al. [1] is noteworthy, as it is well known the presence of chronotropic incompetence of PAH patients [4]. 